\title{
A Comparison of Chat and Audio in Media Rich Environments
}

\author{
Jeremiah Scholl \\ Luleå University of Technology \& \\ Norwegian Centre for Telemedicine \\ Jeremiah.Scholl@telemed.no
}

\author{
John McCarthy \\ University College London \\ Department of Computer Science \\ j.mccarthy@cs.ucl.ac.uk
}

\author{
Rikard Harr \\ Umeå University \\ Department of Informatics \\ rikard.harr@informatik.umu.se
}

\begin{abstract}
This paper presents two case studies of informal group communication using multimedia conferencing that supports various media including video, audio and chat. The studies provide a comparison of audio and chat as communication medium and present data on usage patterns, user preferences and attitudes. The quantitative and qualitative data collected suggest that chat does have advantages in some situations when used for informal communication along with video. The results provide evidence against the hypothesis that chat is a low bandwidth alternative only used when audio communication is unavailable. This suggests that video mediated chat deserves further attention from designers and the research community, since it is often ignored as a "useful" scenario
\end{abstract}

\section{Categories and Subject Descriptors}

H.3.3 [Communications Applications]: Computer conferencing, teleconferencing and videoconferencing. H.5.5 [Group and Organization Interfaces] Computer-supported cooperative work.

\section{General Terms}

Performance, Experimentation, Human Factors.

\section{Keywords}

Chat, video conferencing, collaboration.

\section{INTRODUCTION}

For decades video conferencing has been heralded as a revolutionary technology that would save companies money, reduce energy consumption and relieve overcrowding in urban areas by reducing the need for work related travel [12]. A common reason cited for its failure to live up to expectations was that video conferencing traditionally only supported communication from a few dedicated conferencing rooms, which cut out the possibility of supporting informal communication [12].

Informal communication is associated with a number of characteristics, such as being unscheduled, between random participants, with an unarranged agenda and interactive [13].

Permission to make digital or hard copies of all or part of this work for personal or classroom use is granted without fee provided that copies are not made or distributed for profit or commercial advantage and that copies bear this notice and the full citation on the first page. To copy otherwise, or republish, to post on servers or to redistribute to lists, requires prior specific permission and/or a fee.

CSCW'06, November 4-8, 2006, Banff, Alberta, Canada.

Copyright 2006 ACM 1-59593-249-6/06/0011...\$5.00.
Support for informal communication in group communication systems is viewed as critical since it has been reported to account for over $30 \%$ of total work time, with over $90 \%$ of this time being spent on unplanned conversations [23, 40].

Technological advances now make it possible to deliver video conferencing directly to users' desktops and this opens up a variety of possibilities including support for informal video communication [14]. The creation of new media has also made it possible to compliment audio and video with a variety of communication tools including chat, shared whiteboards, and advanced information visualization. Synchronous text based communication in the form of chat and Instant Messaging (IM) is one such new media that is seeing widespread standalone use in the workplace $[17,19,21,28]$. Despite generally being viewed as a "media-poor" [33] form of communication chat and IM have shown to be effective for supporting spontaneous communication $[17,26]$, and have advocates that view them as underappreciated and underused media [26].

One question surrounding the usage of chat in the workplace is whether it offers its own advantages as a communication medium, or whether it has gained popularity simply because it is easier to install and set-up than audio communication (i.e. low bandwidth, no microphones.). If the latter is the case then it is expected that chat usage will be sparse in media-rich environments where there are no technical barriers to audio communication. However, if the former is the case then chat will continue to be popular even when audio communication is readily available.

This paper compares chat and audio usage within multimedia conferencing systems and presents two Case Studies of informal group communication in a naturalistic setting. In each study, users had a variety of communication media available to them, including video, audio and chat. Qualitative and quantitative data from the groups is presented that supports the conclusion that, contrary to conventional wisdom, chat is a useful medium for communication in media rich environments, at least in some situations. Therefore, chat will not become obsolete as audio/video becomes more widely available.

The rest of this paper is organized as follows. Background studies are presented in section 2. This is followed by the two case studies in section 3. The first case study is of workplace users participating in a "virtual shared office" [14], and the second case study focuses on an educational setting where students are provided with tutoring via a multimedia conferencing tool instead of having an instructor physically available. Conclusions are presented in section 4 and a discussion of further work is presented in section 5 . 


\section{BACKGROUND}

For nearly a century a goal of telecommunication research has been to build a technological infrastructure that enables "face-toface" interaction in distributed settings, to give users a sense of "being there" $[20,39]$ as exemplified by the following quote.

"If as it is said to be not unlikely in the near future, the principle of sight is applied to the telephone as well as that of sound, earth will be in truth a paradise, and distance will lose its enchantment by being abolished altogether." - Arthur Mee 1898 [25]

Originally the "bandwidth hypothesis" claimed that closer semblance between the set of modes that is offered by a technology to those of face-to-face communication the better efficiency is achieved by the use of that technology [38, 42]. Previous research however, has established that there are situations in which low bandwidth media is just as effective as high bandwidth media [7, 8].

Hollan and Stornetta [20] set an alternative goal, "identifying needs which are not ideally met" in real face-to-face interaction. One such documented need for the modern worker is to manage the multitasking nature of the modern workplace, characterized by involvement in multiple initiatives, teams and projects [15, 31].

The role of spontaneous communication in such organizations has been documented elsewhere $[10,14,40]$. Studies have shown that desktop conferencing (with audio and video only) [14] and technologies such as Instant Messaging, Chat and Media Spaces are very useful for stimulating the flow of such communication in the workplace $[17,26]$. Nardi et al. [26] argued strongly for the use of text-based messaging in distributed organizations, which according to them has been neglected in most media spaces, with some exceptions $[35,41]$.

Another need is to manage the increase in interruptions in the modern workplace due to its communication intensity $[10,29]$. Unfortunately, spontaneous communication causes interruptions [10], which may have disruptive effects $[2,4,9]$. While visual interruptions are reported to be more "distracting" than audible interruptions they are also less "stressful", [27] suggesting that they may be preferred in some settings.

Presence can also regulate interruptions by providing feedback about the activity (e.g. working, on the phone, etc.) of the person to be contacted. Awareness about availability can be achieved implicitly by using workspace awareness tools [16], through video [22] or explicitly, when specified by the user (e.g. I'm away from my desk, I'm busy, etc.). Explicit feedback on state is now a ubiquitous feature of instant messaging (IM) applications.

Various studies have been published that compare the effects of audio, video and chat $[5,32,37]$, with chat often reported as an inferior form of communication. Chat has been reported as less effective for building trust than audio or video [5] and studies have also reported that media preferences follow a general media richness trend for a wide range of tasks, with users preferring video, audio and chat in that order [32]. However, none of these studies looked at chat when used in combination with video.

Often chat is simply viewed as a low-bandwidth communication mode [37], and thus something that would not be used in situations where there is enough bandwidth to support video. If chat communication does offer users some unique benefits then this assumption may prove false since video by itself has been shown to be an effective medium for providing presence [11]. For example, some benefits of video-mediated chat (called video-chat for the rest of this paper) in comparison to chat have already been reported for collaborative learning [36].

Studies have also identified some limitations of audio that chat may help mitigate. Ambient noise for example can make it difficult to communicate over an audio channel [24] and it can also be difficult for audio to support more than a single simultaneous thread since people find it awkward to talk over other people in order to hold side conversations [3].

The case studies presented in the next section look at chat and audio usage in a naturalistic setting where video is readily available. They show that chat does offer users a variety of benefits that can lead to it being the preferred medium in some situations.

\section{CASE STUDIES}

A likely explanation for the fast growth of chat in comparison to richer forms of desktop communication is that it is technically easier to deliver. Although there have been wide gains in computing power, bandwidth and camera technology over the past few decades it is still fairly challenging to deliver real-time audio and video to a large number of concurrent users. Despite these barriers some organizations have begun to adopt the technology.

One of these organizations is Luleå University of Technology (LTU). Our studies look at two user groups within LTU that communicate using the commercially available conferencing tool Marratech [1]. Marratech provides users with a variety of communication options including video, audio, chat, and a shared whiteboard. Users may send chat and audio publicly to the entire group or privately to another user. The first Case Study examines a group using Marratech in a research setting. In the second Case Study a group uses Marratech in an educational setting.

The goal of these studies was to collect data on the relative usage of audio and chat in a media rich environment. A key question was whether chat is still a useful communication medium when audio is readily available as an alternative. Thus, we focused specifically on usage patterns and user preferences for chat and audio in our studies and do not report any data in this paper regarding other media, such as the Marratech whiteboard.

\subsection{Case Study 1: The CDT e-corridor}

Desktop conferencing creates the opportunity for a "virtual shared office" [14], where users provide video of each other throughout their work day and communicate using a variety of media. This provides presence and facilitates informal communication between an ad-hoc group that have implicitly expressed a potential interest in communicating with other group members by joining. This provides advantages for informal group communication since for example, it is much more difficult to communicate with the same set of participants using traditional workplace communication technology such as telephones and email, than it is to reach them via the virtual shared office. In addition, they can also be used to host more formal meetings when appropriate.

The Centre for Distance-spanning Technology (CDT) at LTU has been continuously running a virtual shared office, which they call 
their "e-corridor", for approximately 10 years. Participation in the e-corridor is optional and anyone knowing its URL may enter it via the Internet without password authentication. Such a policy clearly has privacy implications but the current users of the ecorridor do not see this as enough of a drawback to keep them from joining. Figure 1 shows a screen shot of a typical day inside the e-corridor and consists of 15 users sending video with only 4 of these users actually being in front of their computer.

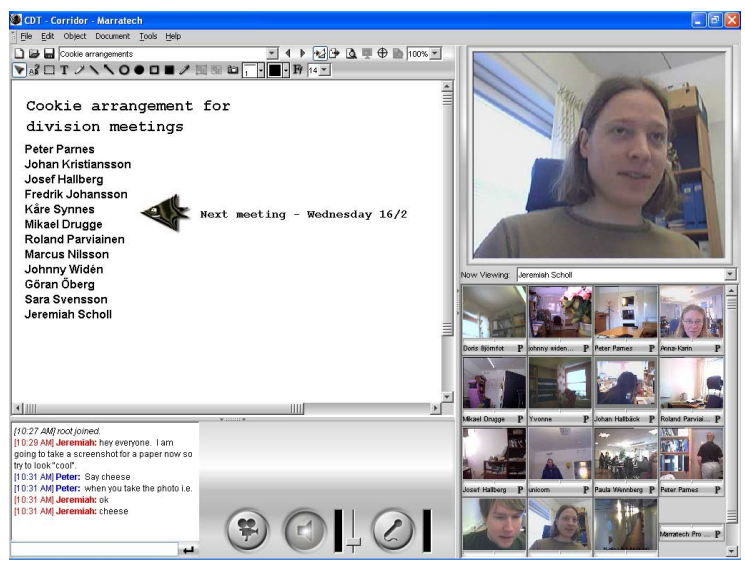

Figure 1: A typical day inside the CDT e-corridor

The e-corridor is visited daily by CDT employees and various project partners including university researchers and administrative staff. The e-corridor was originally set up in the mid 90 s by researchers into IP-Multicast conferencing tools and was based on early MBone tools such as VIC, and locally produced prototypes. Later on as IP-conferencing technology matured it became based on Marratech once it was commercially available. There is a large mix of new and long term users with varying degrees of experience using video conferencing participating in the e-corridor. Today, the e-corridor is based primarily on commercial versions of Marratech but is also augmented with locally produced prototypes, including a History Collection tool [30], which are used to facilitate research into next generation multimedia conferencing tools.

\subsubsection{Method}

Qualitative data was taken from interviews and participatory observations conducted during a previous study on availability management in the e-corridor. The study was conducted by one of the co-authors of this paper, a $\mathrm{PhD}$ Student from another university [j3]. The participatory observations spanned a period of six months. Since the activity in the e-corridor is best described as "bursty" and since private conversations are invisible for a third person, these observations are best described as frequent spot checks. These spot checks were used for the selection of respondents for interviews and for acquiring an understanding of what it is like to be a part of the e-corridor. The interviews that were conducted lasted between 45 and 90 minutes and were taped or recorded. Two of the interviews were conducted in a distributed fashion with Marratech and the others were conducted in facilities at LTU. Quotes and excerpts have been translated from Swedish to English. Results from this study have also been reported in Harr and Wiberg (forthcoming) [18].

Participants (Qualitative)
A total of 10 users from different work categories were selected for interviews. Four project leaders, four project workers and two administrators.

Procedure (Quantitative)

Quantitative data was collected from a database containing events recorded by the History Collection Tool over one academic term (approximately 3 months). In addition to other information, it stores events for each instance of group media usage. Data on private communication is not stored in order to maintain the privacy of e-corridor participants. Usage patterns for related media such as email and telephones were also not collected as the History Collection tool operates solely within Marratech, and because, unlike the media recorded by the History Collection tool, these media are not generally designed or used for communication between an ad-hoc group of participants. The data used in the study was collected previously for the History Collection feature of the e-corridor the users were unaware that the study on media usage was being conducted.

Participants (Quantitative)

The History Collection tool recorded logins by 66 users, 46 of which could be identified by their user name. Of these $80 \%$ (37) were men. This included 9 CDT employees and other university administrative staff, 20 research employees of the university (including $8 \mathrm{Ph} . \mathrm{D}$. students), 2 undergraduates and 14 project partners from affiliated institutions. A questionnaire was conducted by email asking for users' age, experience with the ecorridor and how often they participated in the e-corridor from the same building where CDT is located. 26 users responded with an average age of 38. Experience with the e-corridor ranged from 10 years to new users. The users reported using the e-corridor while physically located in the same building as CDT $60 \%$ of the time with $80 \%$ (21) reporting at least some use of the e-corridor while located in the building.

\subsubsection{Results}

\subsubsection{Qualitative Analysis}

Analysis from the interviews and the participatory observations reveals a variety of reasons why users may prefer either chat or audio in varying contexts. A summary of the results for audio/video is given below, followed by a summary of responses related to the use of chat/video.

\subsubsection{The use of the audio}

According to the respondents, the group audio feature is used almost exclusively within the e-corridor for formal meetings. One reason for this limited use might be that several of the users are collocated in the same building on the campus and as a consequence may meet face-to-face when desirable without too much effort. One participant commented on this issue:

"I find using audio in the e-corridor a little bit uncomfortable as often, most of the people that you see in the e-corridor are also present physically..."

And he continues by saying that:

"If there are many people involved and you feel that you need a more intense discussion then it continues a couple of meters out, then it continues in the corridor, that happens more often. The cost is so small... and the quality is so much better than if everyone sits in their room with their mike and headset." 
The quality aspect that he refers to includes both visual and audio aspects but mostly social cues such as turn taking and floor control. Another user commented like this on his usage of audio/video:

"The only times when I use audio is in those meetings (formal meetings), otherwise we always write."

The formal meetings that do take place in the e-corridor often involve remote participants and are therefore held in the ecorridor with support of video/audio as well as other media. One respondent commented that his usage of the audio function outside formal meetings was very limited.

"It is not that often that I put on my headset and start talking to people. If what I have to say is a little longer, that is faster said, than written, I could imagine doing it. I write pretty fast, so usually it works to write."

However, there are situations where private audio is used for conversation even with co-located colleagues, often for an extended dyadic discussion. This is almost exclusively initiated through a request in the chat channel. The request may consist of a short phrase such as "I am sending audio", just "audio" or "ping" (waiting for the reply "pong"). This is because users don't always wear their headsets and as a consequence will not hear the invitation to the conversation.

The private audio is also used as a substitute for the phone when interacting with a colleague that is remotely located. One user described his use of the audio like this:

"My supervisor lives in Stockholm and I sometimes need to contact him. Instead of contacting him on the phone I contact him through the e-corridor."

\subsubsection{The use of chat}

The main benefits experienced in the use of the chat within the ecorridor as expressed by the respondents are increased awareness, less obtrusion, allowance of intermittent communication and social reasons. Many of these attributes are similar to those reported in other studies of chat in the workplace [6].

\section{Increased awareness}

Another experienced benefit of chat is that it serves a function of an awareness tool. Since it is possible to backtrack the discussion that goes on in chat, it is also used as a tool for recovering after a temporarily absence i.e. achieving awareness based upon asynchronous information, what people have done or said. One user commented on the awareness function of the chat:

"If you follow the discussions that take place there, regardless if you are the sender or the receiver, you still take part of it and achieve an improved picture of what people are doing."

This is an experience that is shared by all our respondents even those that consider themselves as less active in the e-corridor.

\section{Less intrusion}

A general opinion among the respondents is that the chat is experienced as less intrusive than other medium including audio, not only for the intended recipient but also for other users as well, since a textual message displayed in the graphical user interface is considered a lot less disturbing than an audio message. One user commented like this on the question how he would approach a colleague that he estimates as being busy:
“... someone that seems busier, but busy by the computer, I think I would use the public chat. In the public chat everyone is expecting things to happen and won't be very disturbed if it (the chat message, our comment) lies on top".

The ability to communicate with minimal interruption has been attributed to chat in other studies [6]. This suggests that chat may be a preferred medium in some informal contexts since "low personal cost" is feature attributed with its support [13]. Another user said:

"It is a less disruptive interruption to ask a question via an asynchronous medium, they can choose to ignore it while it is much more difficult to ignore someone that is standing in the door way..."

The lack of intrusion also means that a discussion concerning a certain issue could be dealt with in the chat even though it only concerned some participants in the e-corridor and not all. One user commented like this:

"If I do want to talk to a couple of people then I do it in the public chat and they join, those who are not interested will have to ignore it."

This behavior was not considered as negative among the respondents that participated in our study.

\section{Allows asynchronous communication}

As presented above, chat is used for both synchronous and asynchronous communication in the e-corridor, ranging from communicating status such as "gone for lunch" to fast, short and time dependent questions such as "sound?" The use of chat has a tendency to vary from one situation to another, from almost synchronous turn-taking to asynchronous.

"Communication can take place with a lot of stop and go"

This means that it is possible to send a message to someone even though they are considered as busy and as soon as they are idle a response could be expected. A user commented on this issue:

"You just ignore it if you do not want to answer it directly and then it (the message: our comment) stays there as a reminder. I think it is okay if I am in a meeting and then I notice that I have received something, but you do not have to respond until after the meeting, if you have time. "

This might be a beneficial characteristic for a medium in an interaction intensive organization.

\section{Social reasons}

As stated earlier in the paper, a large part of communication in many work environments is spontaneous/informal communication. According to our interviews the main function of chat is as a medium for informal communication among the staff. This may explain why chat is used more frequently than audio, as shown by the quantitative data presented later.

One user commented on this issue:

"We have a constant flow or chatting during the day as you see something you may send something... if you want to go to lunch you write lunch in the public chat and a question mark and then those who wants to go to lunch will respond."

The social nature of chat content was reported by several respondents who commented on the circulation of jokes and the pleasure of being able to see the reaction on people's faces when 
they read a humorous message. This illustrates one way in which the users of the e-corridor compliment chat usage with the video channel. One respondent said concerning this issue:

"It is fun to send something funny to people, then you can see them laugh as they read it."

Another example of how video and chat is intertwined is presented below. In the excerpts the names of the involved actors have been altered in order to secure their integrity and the conversations have been translated into English.

15:19:16.0 Jerry (project leader): Afternoon exercises, Piotr?

15:19:19.0 Jerry: (-:

15:19:24.0 Piotr (project leader): have to stretch once in a while

More work-oriented discussions also take place on a daily basis and the drifting of such discussions may accelerate as more and more participants decide to join. One user said during the interview that a posted question might generate several answers from different respondents.

According to the respondents and to our observations video is an important feature in the e-corridor. Video is sometimes used for keeping track of objects, but mostly serves as a tool for keeping track of people. This may affect audio, chat and other workplace conversations. A few examples of this are given below in order to illustrate how video may compliment chat communication, as this has been much less reported than its use with audio.

One example occurred when the project leader "Karl" was trying to locate his colleague project leader "Ronald" through the video view. In the excerpt some distracting contributions in the sequence have been removed in order to provide clarity.

\section{5:51:47 Karl (project leader): Where is Ronald?}

15:53:57 Jerry (project leader): Ronald is sending from the demo studio. Looks like the sofas there.

This shows how video is used to locate colleagues and also how the public chat is used for short questions and answers. As mentioned above, the video is sometimes also used for keeping track of objects. In the following excerpt four people are discussing a missing delivery in the public chat. Some irrelevant contributions in the sequence have been removed.

14:45:03.0 Piotr (project leader): G: has the package arrived?

14:46:35.0 Jerry (project leader): doesn't look like it in the camera, haven't checked in there. Since I am taking it easy... (-:

14:47:03.0 Piotr: if it hasn't arrived by now you should start looking

14:47:08.0 Piotr: either in my office or mail box maybe

14:47:08.0 Fred (project worker): what are you waiting for?

14:48:15.0 Fred: A [delivery]-guy threw something into Marcus room today

14:48:36.0 Martin (project worker): computer, maybe? :)

14:48:58.0 Fred: small in that case, $<$ A4

14:49:47.0 Piotr: Martin you ought to send video from your office so you can see if something arrives

As shown above, video is used in a variety of ways within the ecorridor and that this does effect chat conversations even though the combination of chat and video is not as "natural" as the combination of audio and video.

\subsubsection{Quantitative Analysis}

An analysis of chat and audio usage recorded by the history tool reveals some interesting patterns that compliment some of the comments made by users during the interviews. During the term at least one of the two modes of communication was reported on 82 separate days. There were 46 users that used either chat or audio at least once.

Analyzing the number of days each of the 46 users communicated with chat and audio using a paired sample Wilcoxon test revealed a significant preference for chat usage $(Z=-3.95, P<0001)$. The preference for chat is illustrated in Figure 2, which shows the number of days each user used the two media. Although around half the sample have a very low usage of both types of media for the other half chat communication is used on many more days than audio communication.

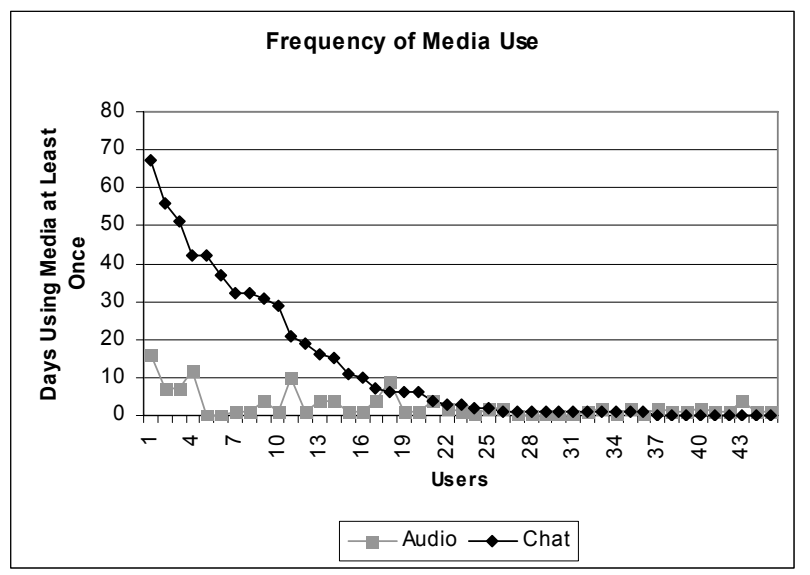

Figure 2: The number of days users communicated with chat and audio usage in the e-corridor.

67.07 chat messages per day were sent in the e-corridor. The top 10 ten users accounted for 60.18 messages with the most active user accounting for 21.66 messages. A larger number of infrequent users resulted in active chat users sending on average only 2.6 chat messages per day. This is not unexpected however because previous studies of workplace chat have reported usage consistently below one message per active user per day [17].

The fact that some users use both media suggests that there are different contexts in which each media is viewed to be useful. In order to learn more about the referred usage we examined media usage by time of day, averaged across the 82 days of use. Chat messages showed to be more frequent in the morning with the peak rate at around 11:00 a.m. By contrast audio communication appeared more frequent in the afternoon with a peak usage at around 17:00 hours.

Further investigation of these peaks in usage indicates that the audio peak at 17.00 is an anomaly caused by two very long audio conferences at 17:00 on two days. However, the peak at 11:00 for chat sessions appears to be a consistent feature. Significant chat traffic is observed at this time on 44 of the 82 days.

These observations are reinforced by the interviews with ecorridor users. The peak at 11:00 can partly be explained by people using the chat function to invite each other to lunch. 


\subsection{Case Study 2: Remote Tutoring for Course Assignments}

The motivation for this second study was twofold. First, we wanted to gain insight from an alternative user set since the CDT corridor is populated by a somewhat unusual set of expert users. These include a large number of people with many years of experience using multimedia conferencing. Second, we wanted to explore media usage in an alternative context to see if this had effects on users' perceptions of the communication media.

The study was of an educational setting with students enrolled in the course Introduction to Media Technology at LTU that received remote assistance on general course work and assignments via Marratech. Using remote assistance instead of having course instructors physically located in student computer labs is a common and growing occurrence in the computer science department. Many instructors feel they are more productive when tutoring remotely from their office since they can continue with their regular work practice more easily than in a computer lab and only need to interact with students when assistance is requested. It also allows instructors to interact with students while traveling and working from home.

The computer science department accommodates this process by hosting a Marratech Manager used for setting up course "emeeting rooms" and also by making Marratech, webcams and headsets available on 35 computers in the student computer labs. During the course the labs were equiped with Marratech version 4.1, 19" Nokia 920C monitors, Sennheiser m@b 30 headsets and Logitech QuickCam Express cameras. Access to the e-meeting rooms is not limited to these labs however as they can also be accessed from anywhere on the Internet.

The course required students to complete several assignments on a computer, such as creating a course homepage and doing a small amount of programming. The student computer labs with cameras and headsets were reserved for students enrolled in the course for approximately 6 hours each week. Participation in the e-corridor was not mandatory but students were encouraged to do so. The instructors pointed out that one advantage of using Marratech was that it allowed students to help each other with questions about assignments, since they could contact each other as well as the instructor, even when working from home. The instructors informed the students that they would be available within the "course e-corridor" for at least 3 hours each week during reserved lab times and that they would try to "hang out" in the e-corridor as much as possible at other times. In general the instructors tried to create a casual and relaxed atmosphere inside the e-corridor.

\subsubsection{Method}

The original course plan included remote assistance via Marratech as described above so the study was conducted with minimal changes to the course plan. At the end of the course a questionnaire was handed out to students during a scheduled lecture that was also used for the general course evaluation. Attendance was mandatory, but filling out the questionnaire was optional. A primary purpose of the questionnaire was to get feedback from students on the remote tutoring process, as it is now becoming the de facto standard in many courses. In order to further investigations into the relative use of audio and chat, questions were added regarding perceptions on how "useful" users found the media to be. The students were asked to rate their agreement on a 7 point Likert scale (strongly agree - strongly disagree) to the statements listed below.

1. E-meetings were a useful tool for gaining information and help during the course.

2. I found group audio to be a useful tool for communication.

3. I found group chat to be a useful tool for communication

4. I found private chat (instant messaging) to be a useful tool for communication

5. I found private audio to be a useful tool for communication.

The following question was also included in order to gain qualitative comments about why users might prefer one medium or the other.

If only one of the following media was provided in combination with video which one would you prefer?

\section{(CHAT) (AUDIO)}

Why?

Of the 105 students enrolled in the course, 82 responded to the questionnaire. Demographic information was collected regarding the students' age, sex and previous experience with related technologies including Chat and IM, VoIP, webcams and Marratech. The average age of the respondents was 21.4 years old, $82 \%$ were male, $96 \%$ had previous experience with chat or IM, $60 \%$ had previously used VoIP, $29 \%$ had used a webcam, and only $6 \%$ had used Marratech.

\subsubsection{Results of the survey}

When given a forced choice between the two media types more students said that they preferred chat over audio: 48 (60\%) vs. 32 $(40 \%)$. However, this bias for chat was not significant. $\left[\chi^{2}(1)=3 . .2, \mathrm{p}=0.07\right.$. ].

Clearer differences were obtained from the ratings of usefulness of the two media types. These were analyzed using a $2 \times 2 \times 2$ mixed ANOVA with Media Type, Communication Type (Public, Private) and Sex as the main factors. Overall the students rated chat as more useful than audio across both public and private $(1: 1)$ communication $[\mathrm{F}(1,79)=48, \mathrm{p}<0.001]$.

These results are illustrated in Figure 3 which shows responses to questions 2 and 3 on the left hand side of the graph and responses to questions 5 and 4 on the right hand side of the graph. A rating of 7 indicates that students strongly agree that the media is useful. A rating of 4 indicates that the students have no opinion on media utility. The rating for public audio use is not significantly different from $4(\mathrm{t}<1, \mathrm{p}=0.939)$.

Ratings across both Media Types were also significantly higher for private communication over public communication $[\mathrm{F}(1,79)=$ $4.74, p<0.05]$ although there was no main effect of interactions with the Sex of the participant.

The qualitative comments collected on why users prefer a particular media revealed a variety of reasons why someone might prefer one mode of communication over the other. Sometimes the students gave reasons that were similar to the reflections of e- 
corridor participants but a number of new and interesting explanations were also given

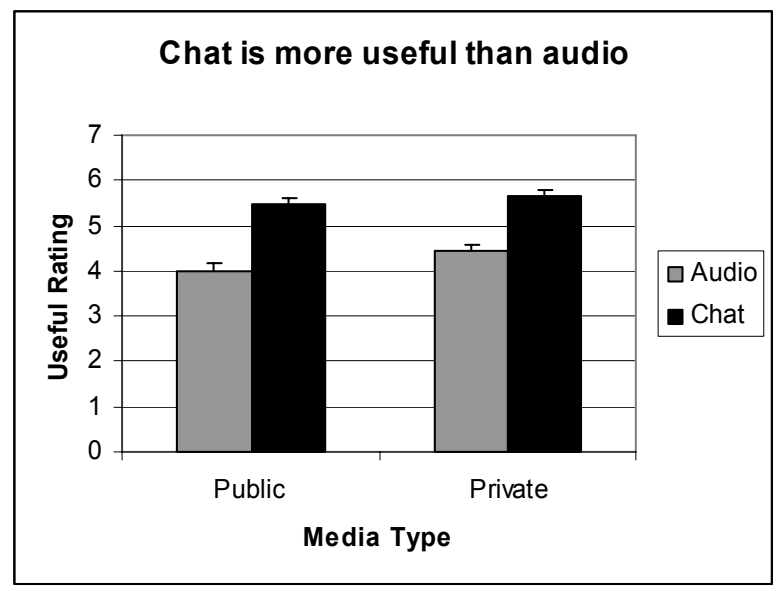

Figure 3: Mean ratings of chat vs. audio.

\subsubsection{Quantitative results}

\subsubsection{Reasons for preferring audio.}

As expected users that preferred audio generally gave responses relating audio as a medium that was easier to use, and that allows for more natural or real communication than chat.

\section{Easier to use}

A few responses related to ease of use were ...

"You don't have to spell words!"

"It's easier to communicate this way"

"well, if you have visual contact in real time it would be just as easy to add audio. That would be more efficient than chat."

"it takes less time to say something than to type it"

\section{Ease of expression}

Comments related to ease of expression included...

"easier to communicate feelings than writing smiley's"

"I really think that only a few feelings are expressed by chat"

"Because with audio and video you can communicate freely and people would understand you better"

"its much easier describing things with audio and when chatting, webcam seems unimportant"

"you get faster feedback"

\section{That natural feeling}

Finally, many users responded that audio simply feels more natural and is better at emulating a real experience.

\footnotetext{
"more fun, gives more of a 'real' feeling"

"better, you get a feeling of the other one feels"

"I like personal contact and audio is better"

"Because it seems more like talking to a person and you get a direct response"
}

\subsubsection{Reasons for preferring chat.}

Respondents that preferred chat cited a variety of reasons. Some of these reasons point to inherent features of chat that lead to it being preferred, whereas others suggest specific scenarios where chat can be useful for overcoming audio problems.

\section{Supports Asynchronous Communication}

One common theme that appeared was that, (similar to Case Study 1) the students liked the asynchronous aspect of chat communication. The asynchronous advantage of chat was stated explicitly in a few responses, and indirectly affected a variety of other responses. Some explicit responses are given below.

"The text stays"

"Easier to keep track of messages"

"Its easier to save the text and look back at it than saving the audio"

One benefit of asynchronous communication is that gives someone the opportunity to think out an answer before responding. Several participants commented that they preferred chat for this reason.

"I like the way I can think for a second before answering"

"You can choose when you want to answer and you can think for a while about the answer"

"Because you can correct what you have written"

\section{Ease of expression}

As discussed earlier some students felt that it was easier to express themselves over the audio channel. Interestingly, many users had similar feelings about chat. One issue that was explicitly mentioned by several students was that the primary language used in the course was English, which was not their first language.

"Its easier to type than speak, in english"

"Audio can be harder to understand, different dialect."

"You don't have to listen to the numbingly bad english"

Some comments mentioned above pointed to the slower pace of chat as one of its advantages and this may be less of an advantage (or even a drawback) when communicating in a native language. The total effect of this factor on the quantitative results are unclear. There were several comments however suggesting that at least some participants would generally prefer chat over audio anyway. One reason for this may be that text based communication has become increasingly common among young people, with technologies such as chat, IM and mobile text messaging leading the way. This is reflected by the demographic data that showed $81(96 \%)$ of the respondents to have had previous experience with either chat or IM. 66 (79\%) respondents marked that they use this technology on a daily basis, with an additional $10(12 \%)$ noting that they use the technology more than once a week.

Some comments related to ease of expression that explicitly mentioned a preference for typing over speaking were ...

"It comes more natural to me since I chat more than I use audio (like telephones)"

"I do this daily at home and am used to it"

"more used to chat really (besides phones that is)"

"Its easier to type in what you feel than actually say it" 


\section{Easier to communicate with a large group.}

One well known problem in video conferencing is that it becomes increasingly difficult to coordinate an audio channel as the number of participants in the session increases. This has led to research into floor control and other turn taking mechanisms. Formal turn taking may be appropriate in some settings but it has the drawback that it hinders informal and impromptu communication, since users must wait their turn before making comments.

Marratech does not provide floor control and several participants commented that it was easier to deal with large groups in chat than with audio. The ease of coordinating a chat channel may be due to its asynchronous aspect, since users can look back at what others have written in order to pay attention to several people at the same time [28].

"easier to chat with more people at the same time"

"If you talk to more than one or two person then audio will be confusing"

"audio gets really messy if you are many"

\subsubsection{Difficulty with audio quality in public places}

Several respondents commented that they prefer chat, not necessarily because of its own merits, but rather because of issues related to audio quality. Several students suggested that audio was "messy" but it is not clear if this was due to difficulty coordinating the audio channel, or because of technical problems.

A few general problems with audio were mentioned, which most likely were a result of the set up in the computer lab. A few of these comments were ...

"because some times the audio can be screwed up"

"because the sound quality is so bad it is hard to hear what people are saying"

"the audio sound was really crappy in the lab"

Poor audio quality in the computer labs is noticed from time to time by course instructors. Often the problem occurs because of difficulties supporting audio from several individual computers at the same location. The student computer labs can be a noisy place, with a lot of people typing, socializing and just passing through. This creates ambient noise that can affect audio usability.

During the beginning of the course it was noticed by the instructors that audio feedback was being created due to some students joining the session from adjacent computers, with their microphone input turned to high levels. This problem was corrected, but audio quality in general seemed to be questionable at times when several students attempted to use audio at the same time while the lab was busier than normal.

Several students also responded that they felt audio was "laggy" or "delayed". It is unlikely that this delay was due to network issues, since participants were generally located within the campus network and the instructors did not notice any problems with network delay while tutoring. A more likely explanation is that even small amounts of delay, that are not noticeable from a remote location, can be quite noticeable when an individual can hear both the live and network transmitted voice of someone speaking at the same time. An additional factor that might have created a perception of delay is related to coordination of the audio channel between various members, since individuals can respond slowly when they are unsure it is their turn to speak.

Many of these specific problems related to audio quality are avoidable in a typical office setting, like the e-corridor, but should occur more frequently in settings where ambient noise is a factor, such as a noisy computer lab.

Privacy

A final comment made by one user that preferred chat was "You get more 'privacy",', which is also an advantage that is specific to public settings. Chat does not allow co-located individuals that are not participating in the conference to listen in on what you are saying (without peering over your shoulder).'

\section{CONCLUSIONS}

The assumption that users would use a richer mode of communication if it were readily available was not supported. In a media rich environment supporting both audio and chat alongside a video channel more users preferred chat to audio and found chat to be more useful than audio for both private and public communication. Specific situational factors, such as communication using a second language or from a semi-public place with a high degree of ambient noise may have affected some of the quantitative results so it is unclear if a general preference for chat over audio will be prevalent among other groups. However, when given a free choice of communication medium, video-chat is expected to be widely used in at least some informal communication environments.

Qualitative comments collected from users in both Case Studies provide insight into why users may prefer chat and suggest that chat does indeed have advantages over audio in some situations.

Some of the advantages of chat that were mentioned by participants in the case studies are.

- It can enable asynchronous communication

- It can lower the "cost" of interrupting others.

- It can help overcome audio problems, such as those that occur in public places.

- It can make it easier to communicate with larger groups.

- It can make it easier to communicate in a second language.

Some of these points have been noted in previous studies of textbased communication [6] - but there have been no previous studies of chat use alongside a video channel, nor when group audio was an option for communicating. The addition of video can augment chat in a number of respects, including:

- An rich indication of presence

- $\quad$ Rich feedback on state or activity

- Indication of involvement in the chat, e.g. reading.

- Feedback on people's emotional reactions.

These factors along with the observation that chat is widely viewed as a useful medium in our Case Studies when used alongside audio and video present a compelling argument that chat will not become an obsolete form of communication in media rich environments. 


\section{FURTHER WORK}

One conclusion from these studies is that video-chat usage is likely to increase in the future as more and more organizations adopt multimedia conferencing as a tool for supporting informal communication. This is already reflected by the growing use of video and IM with home users.

The fact that video-chat is a useful medium presents some interesting directions for future research. In particular research into how to more effectively support video-chat in multimedia applications seems prudent.

Studies that examine video-chat and audio/video in a broader communication context, instead of only comparing their use to each other would also be valuable. Studies of this type have been published on desktop conferencing (without chat) [34] and for chat and IM (without video) [17, 19] [6]. However, this has not been conducted for systems where these media are used in combination with each other.

\section{ACKNOWLEDGMENTS}

This work was done within the VITAL Community project, which is supported by the Objective 1 Norra Norrland - EU structural fund programme for Norra Norrland. Support was also provided by the Centre for Distance-spanning Technology (CDT). Thank you to Rolland Parviainen for providing the usage of his history collection tool and to Peter Parnes for help proofreading.

\section{REFERENCES}

[1] Marratech AB. http://www.marratech.com

[2] Adamczyk, P.D. and Bailey, B.P., If not now, when?: The effects of interruption at different moments within task execution. in Proceedings of the SIGCHI conference on Human factors in computing systems (CHI '04), (Vienna, Austria, April 24 - 29, 2004), ACM Press, New York, NY, 271-278.

[3] Aoki, P.M., Romaine, M., Szymanski, M.H., Thornton, J.D., Wilson, D. and Woodruff, A., The Mad Hatter's Cocktail Party: A Social Mobile Audio Space Supporting Multiple Simultaneous Conversations. in Proceedings of the SIGCHI conference on Human factors in computing systems (CHI '03), (Fort Lauderdale, Florida, 2003), ACM Press, New York, NY, 425-432.

[4] Bailey, B.P., Konstan, J.A. and Carlis, J.V., Measuring the effects of interruptions on task performance in the user interface. in Proceedings of the IEEE Conference on Systems, Man, and Cybernetics (SMC '00), (Nashville, TN, Oct. 8-11, 2000), IEEE, 757-762.

[5] Bos, N., Gergle, D., Olson, J.S. and Olson, G.M., Being there versus seeing there: Trust via video. in Proceedings of the SIGCHI conference on Human factors in computing systems, Ext. Abstracts (CHI '01), (Seattle, WA, April 20 25, 2001), ACM Press, New York, NY, 291 - 292.

[6] Bradner, E., Kellogg, W. and Erickson, T., The Adoption and Use of "Babble": A Field Study of Chat in the Workplace. in Proceedings of the European Conference on Computer Supported Cooperative Work (ECSCW'99), (Copenhagen, Denmark, Sept. 12-16, 1999), Kluwer Academic Publishers, Dordrecht, 139-158.
[7] Chapanis, A., Ochsman, R., Parrish, R. and Weeks, G. Studies in Interactive Communication. 1: The effects of four communication modes on the behaviour of teams during cooperative problem solving. Human Factors, 14, (1972), 487-509.

[8] Chapanis, A., Parrish, R., Ochsman, R. and Weeks, G. Studies in Interactive Communication. II: The effects of four communication modes on the behaviour of teams during cooperative problem solving. Human Factors, 19, 2 (1977), 101-125.

[9] Czerwinski, M., Cutrell, E. and Horvitz, E., Instant Messaging and Interruption: Influence of Task Type on Performance. in Proceedings of OZCHI '00, (Sydney, Australia, Oct. 4 - 8, 2000), 356-361.

[10] Dabbish, L. and Kraut, R.E., Controlling Interruptions: Awareness Displays and Social Motivation for Coordination. in Proceedings of the ACM conference on Computer supported cooperative work (CSCW'04), (Chicago, IL, Nov. 6 - 10, 2004), ACM Press, New York, NY, 182 - 191.

[11] Dourish, P. and Bly, S., Portholes: Supporting Awareness in a Distributed Work Group. in Proceedings of the SIGCHI conference on Human factors in computing systems (CHI '92), (Monterey, CA, May 3 - 7, 1992), ACM Press, New York, NY, 541-547.

[12] Egido, C., Video conferencing as a technology to support group work: a review of its failures. in Proceedings of the ACM conference on Computer supported cooperative work (CSCW '88), (Portland, OR, Sept. 26-28, 1988), ACM Press, New York, NY, 13 - 24.

[13] Fish, R.S., Kraut, R.E. and Chalfonte, B.L., The VideoWindow System in Informal Communication. in Proceedings of the ACM conference on Computer supported cooperative work (CSCW'90), (Los Angeles, CA, October 710, 1990), ACM Press, New York, NY.

[14] Fish, R.S., Kraut, R.E., Root, R.W. and Rice, R.E. Evaluating Video as a Technology for Informal Communication. Communications of the ACM, 36, 1 (1993), $48-67$.

[15] Gonzalez, V.M. and Mark., G., Constant, Constant, Multitasking Craziness: Managing Multiple Working Spheres. in Proceedings of the SIGCHI conference on Human factors in computing systems (CHI '04), (Vienna, Austria, April 24 29, 2004), ACM Press, New York, NY, 113-120.

[16] Gutwin, C. and Greenberg, S., Workspace awareness for groupware. in Proceedings of the SIGCHI conference on Human factors in computing systems, Ext. Abstracts (CHI '01), (Vancouver, British Columbia, Canada, 1996), ACM Press, 208-209.

[17] Handel, M. and Herbsleb, J.D., What Is Chat Doing in the Workplace? in Proceedings of the ACM conference on Computer supported cooperative work (CSCW'02), (New Orleans, Louisiana, Nov. 16-20, 2002), ACM Press, New York, NY, 1 - 10.

[18] Harr, R. and Wiberg, M. Lost in translation: Investigating the ambiguity of availability cues in an online media space. To be published in the Internation Journal Behaviour and Information Technology (BIT). 
[19] Herbsleb, J.D., Atkins, D.L., Boyer, D.G., Handel, M. and Finholt, T.A., Introducing Instant Messaging and Chat in the Workplace. in Proceedings of the SIGCHI conference on Human factors in computing systems (CHI '02), (Minneapolis, Minnesota, April 20-25, 2002), ACM Press, New York, NY, 171 - 178.

[20] Hollan, J. and Stornetta, S., Beyond Being There. in Proceedings of the SIGCHI conference on Human factors in computing systems (CHI '92), (Monterey, CA, May 3-7, 1992), ACM Press, New York, NY, 119 - 125.

[21] Isaacs, E., Walendowski, A., Whittaker, S., Schiano, D.J. and Kamm, C., The character, functions, and styles of instant messaging in the workplace. in Proceedings of Conference on Computer-Supported Cooperative Work (CSCW), (New Orleans, LA, Nov. 16-20, 2002), 11-22.

[22] Johnson, B. and Greenberg, S., Judging People's Availability for Interaction from Video Snapshots. in Proceedings of Hawaii International Conference on System Sciences (HICSS), (Maui, Hawaii, Jan. 5-8, 1999), IEEE Computer Society.

[23] Kraut, R.E., Fish, R., Root, R. and Chalfonte, B. Informal communication in organizations: Form, function, and technology. in Oskamp, S. and Spacapan, S. eds. Human reactions to technology: Claremont symposium on applied social psychology, Sage Publications, Beverly Hills, CA, 1990, 145-199.

[24] Mantei, M.M., Baecker, R.M., Sellen, A.J., Buxton, W.A.S., Milligan, T. and Wellman, B., Experiences in the use of a media space. in Proceedings of the SIGCHI conference on Human factors in computing systems, ( New Orleans, Louisiana, 1991), ACM Press, 203 - 208.

[25] Mee, A. The Pleasure Telephone the Strand Magazine, 1898, 339-369.

[26] Nardi, B.A., Whittaker, S. and Bradner, E., Interaction and outeraction: instant messaging in action. in Proceedings of the ACM conference on Computer supported cooperative work (CSCW'00), (Philadelphia, PA, Dec. 2-6, 2000), ACM Press, New York, NY, 79 - 88.

[27] Nilsson, M., Drugge, M., Liljedahl, U., Synnes, K. and Parnes, P., A Study on Users' Preference on Interruption When Using Wearable Computers and Head Mounted Displays. in Proceedings of IEEE International Conference on Pervasive Computing and Communications (PerCom'05), (Kauai, Hawaii, March 8-12, 2005), 149-158.

[28] O'Neill, J. and Martin, D., Text Chat in Action. in Proceedings of the ACM SIGGROUP conference on Supporting group work (GROUP '03), (Sanibel Island, Florida, November 9-12, 2003), ACM Press, New York, NY, $40-49$.

[29] Panko, R.R. Managerial Communication Patterns. Journal of Organizational Computing, 2, 1 (1992), 95-122.

[30] Parviainen, R. and Parnes, P., A Web Based History tool for Multicast e-Meeting Sessions. in Proceedings of the IEEE
International Conference on Multimedia \& Expo (ICME '04), (Taipei, Taiwan, June 27 - 30, 2004), IEEE.

[31] Rouncefield, M., 'Hughes, J.A., Rodden, T. and Viller, S., Working with "Constant Interruption": CSCW and the Small Office. in Proceedings of the ACM conference on Computer supported cooperative work (CSCW'94), (Chapel Hill, NC, Oct. 22-26, 1994), ACM Press, New York, NY, 275 - 286.

[32] Schliemann, T., Asting, T., Følstad, A. and Heim, J., Medium preference and medium effects in person-person communication. in Proceedings of the SIGCHI conference on Human factors in computing systems, Ext. Abstracts (CHI '02), (Minneapolis, Minnesota, April 20-25, 2002), ACM Press, New York, NY, 710 - 711.

[33] Short, J., Williams, E. and Christie, B. The Social Psychology of Telecommunications. Wiley, New York, 1976.

[34] Tang, J.C. and Isaacs, E.A. Why do users like video? studies of multimedia-supported collaboration. Computer-Supported Cooperative Work: An International Journal, 1, 3 (1993), 163-196.

[35] Tang, J.C., Isaacs, E.A. and Rua, M., Supporting distributed groups with a Montage of lightweight interactions. in Proceedings of the ACM conference on Computer supported cooperative work (CSCW'94), (Chapel Hill, NC, Oct. 22-26, 1994), ACM Press, New York NY, 23 - 34.

[36] Tscholl, M., McCarthy, J.D. and Scholl, J., The effect of video-augmented chat on collaborative learning with cases. in Proceedings of Proceedings of Computer Supported Collaborative Learning (CSCL '05), (Taipei, Taiwan, May 30 - June 4, 2005).

[37] Vera, A., Kvan, T., West, R.L. and Lai, S., Expertise, collaboration and bandwidth. in Proceedings of the SIGCHI conference on Human factors in computing systems (CHI '98), (Los Angeles, CA, Apr. 18 - 23, 1998), ACM Press, New York, NY, 503 - 510.

[38] Whittaker, S. Theories and Methods in Mediated Communication. in Graesser, A., Gernsbacher, M. and Goldman, S. eds. The Handbook of Discourse Processes, Erlbaum, NJ, 2002, 243-286.

[39] Whittaker, S. Things to talk about when talking about things. Human-Computer Interaction, 18, (2003), 149 - 170.

[40] Whittaker, S., Frohlich, D. and Daly-Jones, O., Informal workplace communication: what is it like and how might we support it? in Proceedings of the SIGCHI conference on Human factors in computing systems (CHI '94), (Boston, MA, Apr. 24-28, 1994), ACM Press, New York, NY, 131 137.

[41] Whittaker, S., Swanson, J., Kucan, J. and Sidner, C. TeleNotes: managing lightweight interactions in the desktop. ACM transactions on Computer-Human Interaction (TOCHI), 4, 2 (1997), 137-168.

[42] Williams, E. Experimental comparisons of face-to-face and mediated communication: A Review. Psychological Bulletin, 84, 5 (1977), 963-976. 